\title{
MDCT angiographic findings of various congenital pulmonary artery anomalies in pediatric patients
}

\author{
Mohamed M. Harraz $1^{1 *}$, Ahmed H. Abouissa', Hala A. Saleh², Khalid A. Attas ${ }^{3}$, Samira M. Al-Yamani
} Ganadiel Alsulami ${ }^{3}$ and Mohamed M. Refai ${ }^{3}$

\begin{abstract}
Background: Congenital pulmonary artery anomalies are variable and need proper diagnosis and treatment. CT angiography with multiplanar reconstruction has the main role in the assessment of these anomalies and this noninvasive method should be the method of choice for preoperative planning and postoperative follow up. The aim of the study is to assess the value of MDCT in the detection of pulmonary arteries anomalies in the pediatric population with complex congenital heart disease in conjunction with echocardiography as an alternative to conventional angiography and to determine the superiority of MDCT in the assessment of other abnormalities such as airway anomalies.
\end{abstract}

Results: In our retrospective study, 52 patients (28 male and 24 females, aged 1 day to 4 years: mean age 2 years) were examined with contrast-enhanced CT. CT examinations were done using a 128-section CT scanner (Siemens Somatom Definition AS) using non-ionic iodinated contrast media. 2D and 3D reconstructions were performed. The correlation was made with echocardiograms. All imaging studies were reviewed. The echo was done to all patients. Surgery and/or catheter angiography performed to all patients, their findings were reviewed and compared to CTA findings. Other abnormalities such as congenital airway anomalies are detected using axial MDCT images and reconstructed imaging techniques.

MDCT was accurate in revealing pulmonary artery anomalies. The commonest pulmonary artery anomaly was atresia, stenosis then hypoplasia. These anomalies may be isolated or associated with other congenital heart diseases. In the current study, MDCT could diagnose all cases of pulmonary arterial anomalies with 96\% sensitivity, 100\% specificity, 98\% accuracy, 100\% positive, and 94\% negative predictive values. CT scans provide accurate information to assess complex spatial relationships of vascular airway compression frequently associated with $\mathrm{CHD}$ in the pediatric population.

Conclusion: MDCT scanner can be an alternative to diagnostic conventional angiography for the non-invasive assessment of the pulmonary artery. Higher quality multiplanar and 3D reconstruction achieved by the MDCT scanners offer a rapid, reliable and non-invasive technique that can be used for the evaluation and preoperative assessment of thoracic vascular and extra-vascular anatomy in infants and children with suspected congenital heart disease. CT technologies are constantly developing collaboration between radiologists, pediatric cardiologists, and anesthesiologists, which is essential for improving CT performance.

Keywords: MDCT, Pulmonary arteries, Congenital anomalies, CT angiography

\footnotetext{
* Correspondence: harrazharraz@live.com

'Radiology Department, Mansoura University Hospitals, Mansoura, Egypt

Full list of author information is available at the end of the article
} 


\section{Background}

CT angiography (CTA) is an important modality in the evaluation of pediatric congenital heart disease (CHD). It can be used for the accurate depiction of complex cardiovascular anatomic features both before and after surgery and of a variety of post-treatment complications. CT facilitates the assessment of extracardiac structures. It is important for radiologists to have extensive knowledge of cardiac and extracardiac anatomy and surgical techniques [1].

Although echocardiogram and conventional angiography are usually known as the primary imaging for assessment of CHD, echocardiography may not be enough for evaluating the extracardiac structures, such as great vessels due to acoustic window limitations [2, 3]. Conventional angiography is reduced by its $2 \mathrm{D}$ nature and difficulties in simultaneous evaluation of the systemic and pulmonary vascular systems [3]. Compared with CT, cardiac angiography has more risks because of its invasive nature $[4,5]$, it needs anesthesia and a large volume of contrast injection [4], and more radiation exposure to the patient [1].

Compared with CT in the past, MDCT scanners yield images with better temporal and spatial resolution, greater anatomic coverage, more consistent enhancement with a lesser volume of contrast material injection, and higher-quality 2D and $3 \mathrm{D}$ reconstruction. Fast imaging with these CT scanners needs less patient sedation than older-generation CT and conventional angiography [6]. CT can be used to asses patients with CHD knew or suspected with echocardiogram for which further imaging is needed to characterize extracardiac anomalies before surgery. The pulmonary arteries, pulmonary veins, aortic arch, and great vessels may be inadequately characterized at echocardiography, requiring more diagnosis with CT [3]. CT can be used in the postoperative evaluation of CHD patients who have a variety of suspected treatment-related complications. Because it helps rapid assessment of vascular structures, the lungs, and potential spaces, CT can be used in the preoperative and postoperative evaluation of CHD patients whose condition suddenly deteriorates. In this condition, CT quickly diagnoses a lot of CHD-related complications such as pulmonary embolism, pneumonia, pericardial and pleural effusion. CT can be used before a reoperation to assess altered anatomic features related to previous surgery [7].

Although MRI has no radiation exposure and used for functional assessment of cardiovascular structures, MRI has several limitations compared with CT. These include poorer spatial resolution, image-degrading artifacts from implanted metal, such as intravascular stents and embolization coils expensive, limited availability, contraindication in patients with pacemakers, and increased need for general anesthesia in

Table 1 Associated finding with the pulmonary artery anomalies

\begin{tabular}{|c|c|c|}
\hline Type & Number & Associated finding \\
\hline Pulmonary artery atresia & 16 & \multirow{4}{*}{$\begin{array}{l}\text { PDA, Dextrocardia, Dilated collaterals, overriding aorta, hypoplastic } \\
\text { aortic arch, VSD, Fallot tetralogy }\end{array}$} \\
\hline \multirow{2}{*}{$\begin{array}{l}\text { Pulmonary atresia with ventricular septal defect pulmonary artery } \\
\text { atresia with intact ventricular system }\end{array}$} & 11 & \\
\hline & 4 & \\
\hline Complex pulmonary atresia & 1 & \\
\hline Pulmonary artery stenosis & 13 & \multirow[t]{5}{*}{ PDA, Dextrocardia, Dilated collaterals, overriding aorta, Fallot tetralogy. } \\
\hline Type I & 6 & \\
\hline Type ॥ & 4 & \\
\hline Type III & 3 & \\
\hline Type IV & - & \\
\hline Pulmonary artery hypoplasia & 10 & $\begin{array}{l}\text { PDA, Dextrocardia, Dilated collaterals, overriding aorta, right side } \\
\text { aortic arch, VSD }\end{array}$ \\
\hline Pulmonary artery dilatation & 8 & Dilated aorta, dilated left ventricle, right side aortic arch \\
\hline Aorta-pulmonary anomalies & & Right side aortic arch \\
\hline Truncus arteriosus & 2 & \\
\hline PDA & 27 & \\
\hline Transposition of great vessels & - & \\
\hline \multicolumn{3}{|l|}{ Aberrant artery } \\
\hline Aberrant right pulmonary artery & 3 & Aortic anomalies and PDA \\
\hline Aberrant left pulmonary artery & 2 & Tetralogy of Fallot and anomalies subclavian arteries \\
\hline Aberrant right subclavian artery & 4 & \\
\hline Both aberrant right subclavian artery with left pulmonary artery & 2 & \\
\hline Left pulmonary artery sling & 1 & \\
\hline
\end{tabular}


Table 2 Agreement between catheter/operative data and MDCT for pulmonary anomalies

\begin{tabular}{llllllllcc}
\hline & True & False & True & False & Sensitivity (\%) & Specificity & PPV & NPV (\%) & Accuracy (\%) \\
& Positive & Positive & Negative & Negative & & & & \\
\hline Pulmonary artery atresia & 15 & 0 & 22 & 1 & 94.2 & 100 & 100 & 95.5 & 96.5 \\
Pulmonary artery stenosis & 12 & 0 & 35 & 1 & 92.5 & 100 & 100 & 96.9 & 98.01 \\
Pulmonary artery hypoplasia & 9 & 0 & 15 & 1 & 90 & 100 & 100 & 93.4 & 96.0 \\
Pulmonary artery dilatation & 7 & 0 & 20 & 1 & 90 & 100 & 100 & 94.3 & 96.02 \\
Aorta-pulmonary anomalies & 29 & 0 & 25 & 1 & 97 & 100 & 100 & 95.5 & 98.01 \\
Aberrant artery & 11 & 0 & 16 & 1 & 92 & 100 & 100 & 93.8 & 96.5 \\
Total pulmonary anomalies & 83 & 0 & 133 & 6 & 96 & 100 & 100 & 94 & 98 \\
\hline
\end{tabular}

younger children. MRI also takes a longer time than CT, possibly precluding imaging of critically ill, thermally unstable, and uncooperative pediatric patients. MRI is limited in the evaluation of the airways and lungs structures [8-12].

The pulmonary arteries are involved in most congenital heart lesions, especially in patients with right ventricular outflow tract obstruction. In these patients, precise preoperative delineation of the pulmonary arteries is needed. Outcomes are improved because of innovations in the surgical approach which are influenced by findings from using newer crosssectional imaging techniques. Imaging studies, therefore, play an important role in the treatment of these patients [13].

In our study, we tried to test the efficacy of MDCT machines using a 128-section CT scanner in the diagnosis of
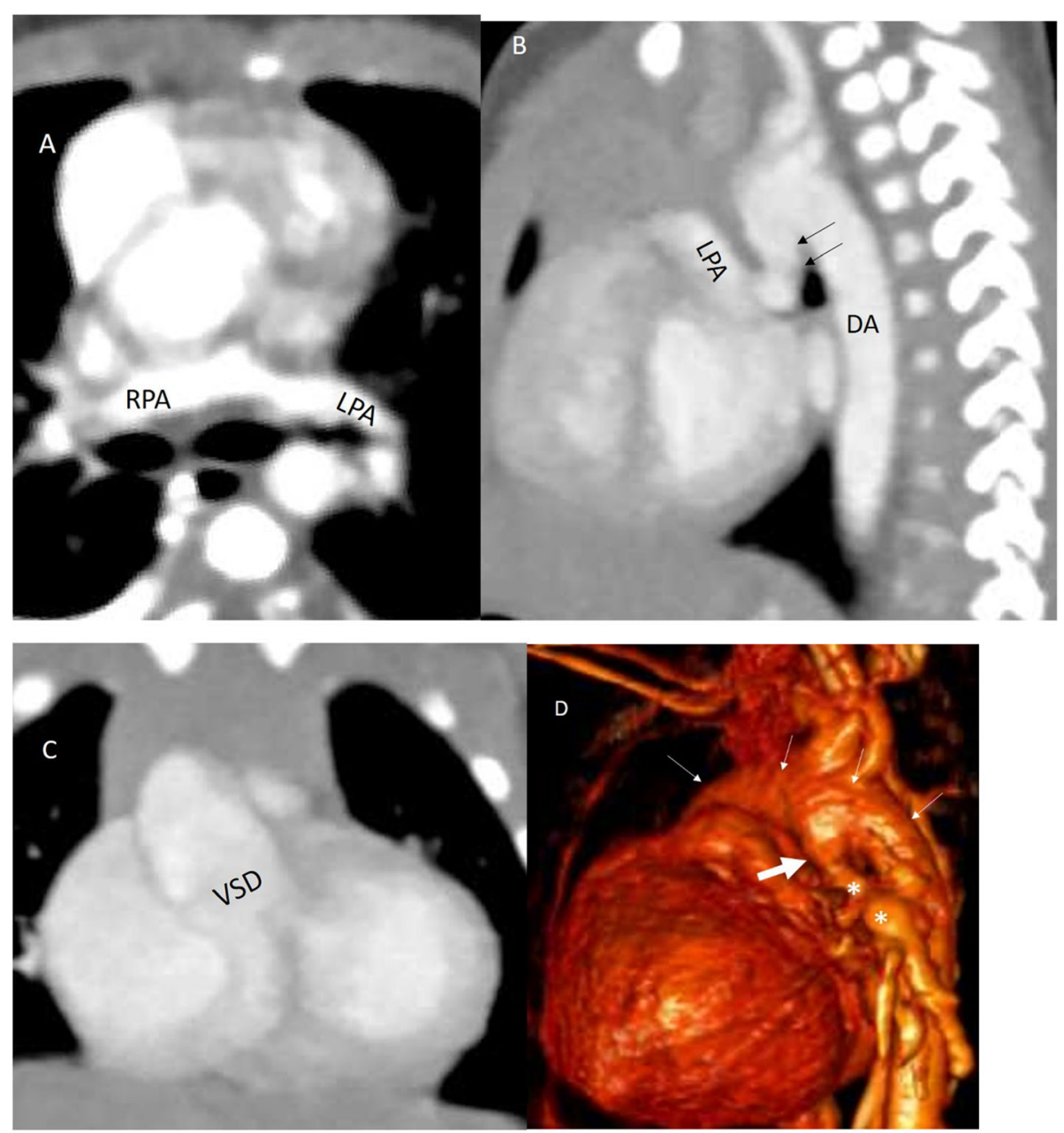

Fig. 1 A 3-week-old baby with pulmonary atresia and low O2 saturation. a Axial CT shows atretic MPA, small RPA, and LPA. b Sagittal 2D MIP: refilling of the LPA via a vertical PDA (arrows). c Coronal 2D MIP shows a large VSD. d 3DVR: vertical PDA (black arrow) arising from aorta (small arrows) to supply the LPA (*) 


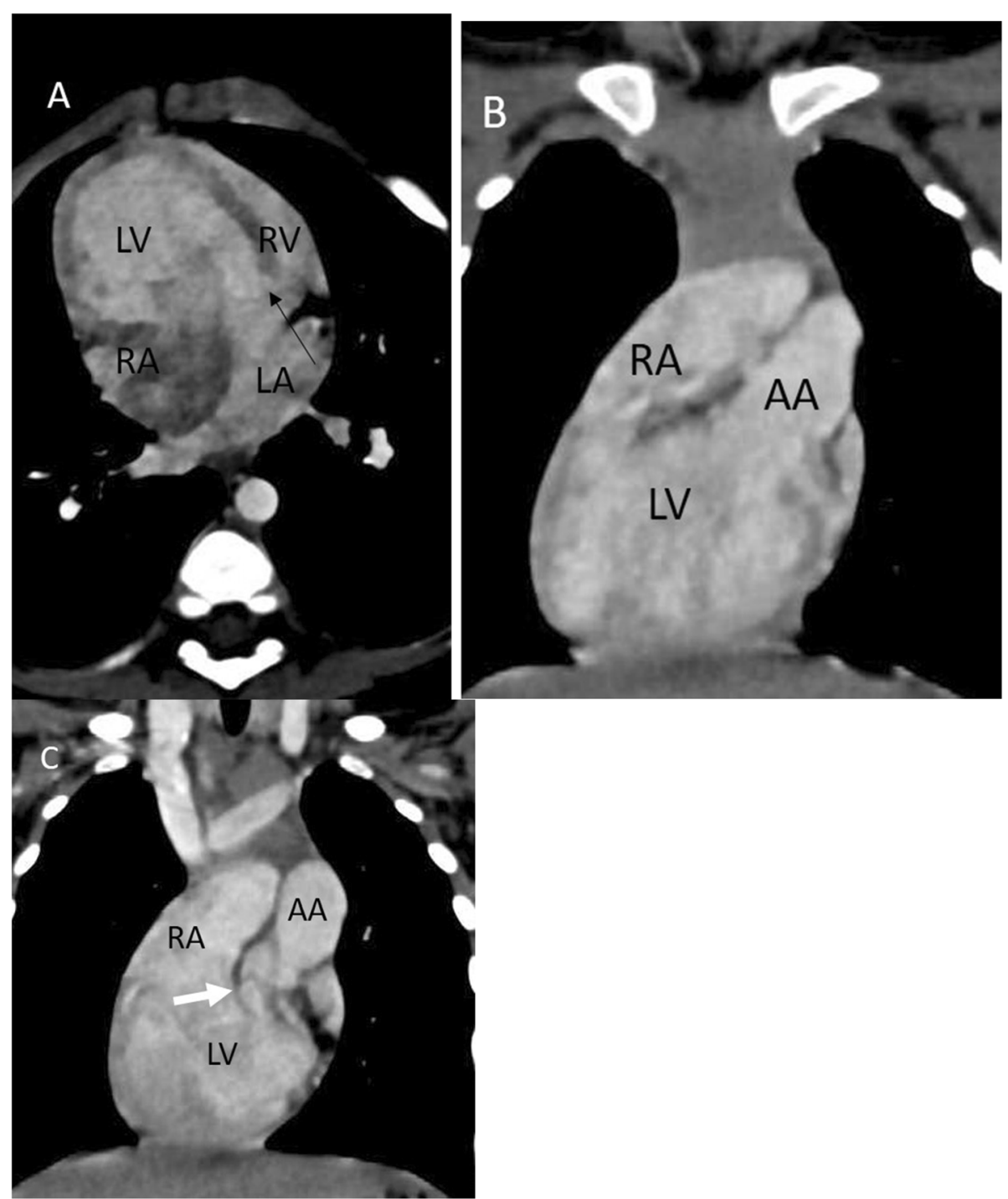

Fig. 2 A 2-year-old boy presented with respiratory distress and low O2 saturation. a Axial CTA shows dextrocardia, hypoplastic RV, dilated double inlet LV receives both atria, and VSD (arrow). b Coronal CTA shows aorta arises from the enlarged LV. c Coronal CTA shows small atretic pulmonary artery(arrow)

pulmonary artery anomalies before surgical planning or therapeutic angiography.

\section{Methods}

In our retrospective study, 52 patients $(28$ males and 24 females, aged 1 day to 4 years: mean age 2 years) who had CHD and possible pulmonary arteries anomalies clinically or by echo, exclusion criteria were all patient with contraindication to contrast injection either due to allergy or impaired renal functions not on dialysis and patients missed their follow-up. All patients were subjected to MDCTA between January 2016 and December 2018. All CT finding correlated with their surgical/or conventional angiography.

\section{CT technique}

Before the baby is received, the CT room was warmed and the light was slightly dimmed. The baby is wrapped well to avoid hypothermia. Sedation with chloral hydrate syrup, $50-75 \mathrm{mg} / \mathrm{kg}$ PO 30-60 min before procedure, may be repeated in $30 \mathrm{~min}$ if necessary, not to exceed $120 \mathrm{mg} / \mathrm{kg}$ or $1 \mathrm{~g} /$ dose in infants or $2 \mathrm{~g} /$ dose in children. Multi-detector CTA examinations were performed with a 128-section CT scanner (Siemens Somatom Definition AS). Patients were examined in a supine position, and all scans were extending from the root of the neck to the aortic bifurcation in cranio-caudal direction to assess abdominal vascular structures and viscera. Non-ionic iodinated contrast material (Omnipaque or Xenetix $350 \mathrm{mgI} / \mathrm{ml}$ ) was injected by a power injector at a rate varying from $0.4 \mathrm{ml} / \mathrm{s}$ to 1 
$\mathrm{ml} / \mathrm{s}$, total dose does not exceed $2 \mathrm{ml} / \mathrm{kg}$ of body weight. Saline injection followed contrast with the same volume and rate. Due to various access, e.g., arm, leg, umbilical, we used a modified bolus tracking method. When contrast is just seen in the upper part of descending aorta, the scan starts. We used this technique to ensure the opacification of the cardiac chambers and great vessels. Those patients have complex anomalies that make optimum timing not guaranteed. Acquisition parameters used for CT examinations were as follows: $80 \mathrm{KVp}, 100-240 \mathrm{mAs}(10 \mathrm{~mA} / \mathrm{kg})$, and $0.6 \mathrm{~mm}$ slice thickness. The gantry rotation time was
$0.3 \mathrm{~s}$, the collimation was $128 \times 0.6 \mathrm{~mm}$ and the matrix was $512 \times 512$. The reconstructed images were $0.75-\mathrm{mm}$ thick with a $0.5 \mathrm{~mm}$ interval. $2 \mathrm{D}$ and $3 \mathrm{D}$ reconstructions in different planes and intensity projections were performed. 3D VR for airways assessment was also performed. CT findings were retrospectively interpreted by two radiologists with 8 and 14 years of experience without knowledge of the image findings of other modalities. Surgery and/or catheter angiography were performed to all patients, and their findings were reviewed and compared to CTA findings.

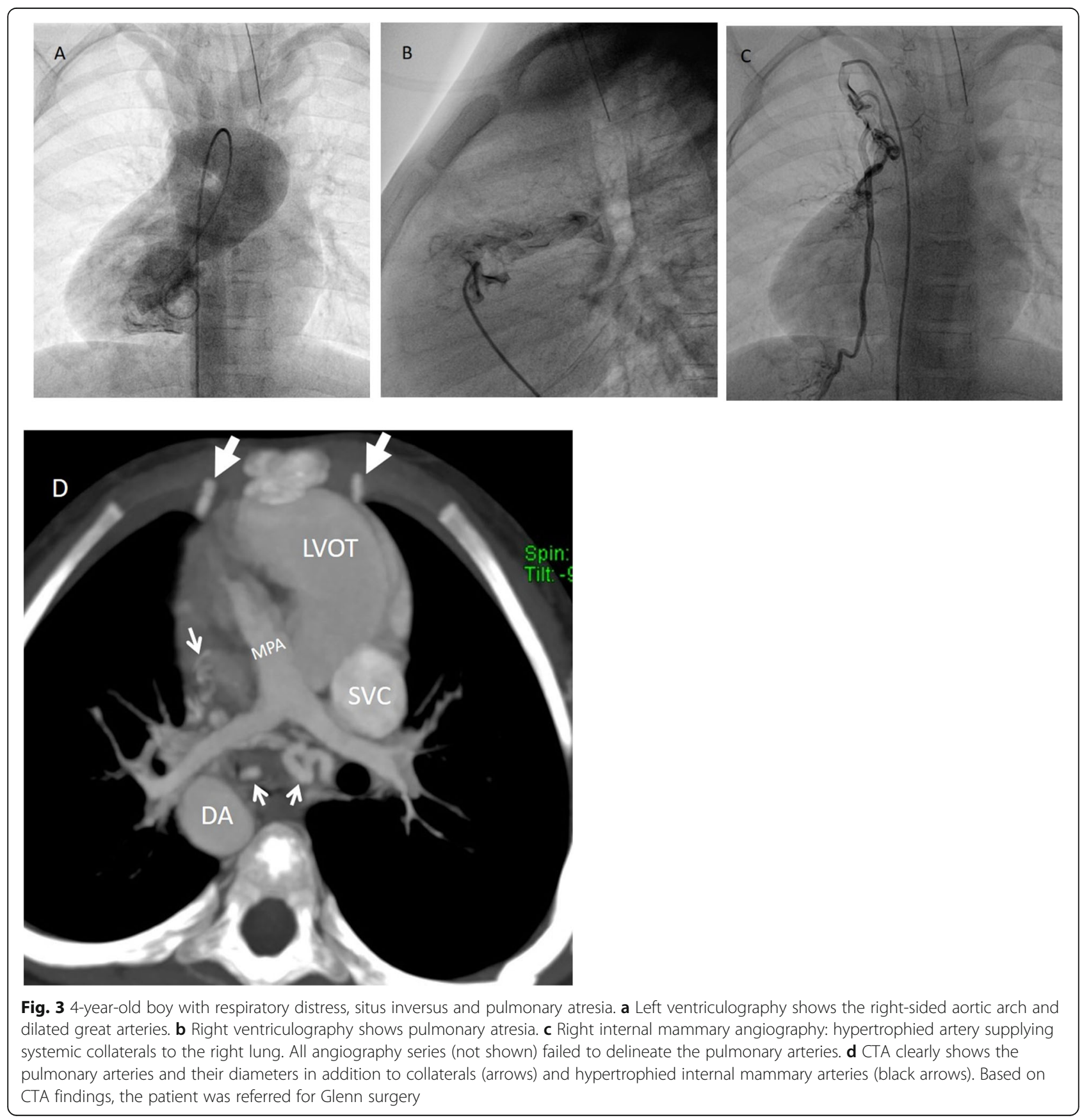




\section{Results}

One hundred and twenty-eight-slice CT scanner was accurate in revealing pulmonary artery anomalies. CT pulmonary angiography was superior to echocardiography in the visualization of the morphology of the pulmonary artery (PA) congenital anomaly and its associated findings. The commonest pulmonary artery anomaly was atresia, stenosis then hypoplasia and dilatation in addition to aortopulmonary artery collateral arteries (Table 1). These anomalies may be isolated or associated with other congenital heart diseases. All cases of pulmonary abnormalities diagnosed on 128-section CT scanner in our study were correlated with echocardiography (all cases), surgery (26 cases), conventional angiography (preoperative diagnosis) 19 cases and 7 cases underwent therapeutic convolutional angiography. In the current study, MDCT could diagnose all cases of pulmonary arterial anomalies with 96\% sensitivity, 100\% specificity, 98\% accuracy, $100 \%$ positive, and $94 \%$ negative predictive values (Table 2). The commonest pulmonary artery anomaly was atresia in 16 cases (30\%) (Figs. 1, 2, and 3). Pulmonary atresia with a ventricular septal defect in 11 cases, pulmonary artery atresia with intact ventricular system 4 cases, and 1 case complex pulmonary atresia. The most common associated finding is PDA, followed by stenosis of pulmonary artery in 13 cases (25\%), 6 cases type I, 4 cases type II, 3 cases type III, and no case in our study found type IV Figure 4 shows type I pulmonary stenosis, then hypoplasia of main pulmonary artery in 10 cases $(20 \%)$ or its main branches (Fig. 1) and pulmonary artery dilatation in 8 cases (Figs. 5 and 6). Some of the anomalies affect both aorta and pulmonary artery (Figs. 4, 5, and 6).
In our study, there was a patent ductus arteriosus (27 cases) (Figs. 1 and 6) and truncus arteriosus (2 cases). In our study, we found aberrant right pulmonary artery (Fig. 5) (3 cases), aberrant left pulmonary artery (Fig. 7) (2 cases), left pulmonary artery sling (1 case), aberrant right subclavian artery (Fig. 6) (4 cases), both aberrant right subclavian artery with left pulmonary artery (Fig. 8) (2 cases). CT can be used for accurate detection of post-treatment complications, in our study we have one case shows fistula between coronary arteries and right ventricle (Fig. 9). CT facilitates the assessment of extracardiac structures as congenital tracheal stenosis (Fig. 8). However, CT angiography was not accurate for detecting valvular diseases of the heart. The intraoperative findings were consistent with the pulmonary CT angiographic findings.

\section{Discussion}

MDCT assesses a lot of congenital pulmonary arterial anomalies, such as atresia, stenosis, hypoplasia, and dilatation in addition to the postoperative appearance of the pulmonary arterial tree. These anomalies may be isolated or associated with other cardiovascular anomalies. CT helps assessment of the central and peripheral pulmonary arteries. In CT study we can detect the presence, patency, confluence, or caliber of the pulmonary arteries which must be diagnosed before definitive surgery as this information can affect surgical decision-making [14]. Pre- and postoperative assessment of the pulmonary artery can be accurately and non-invasively detected with CT. Reformatted
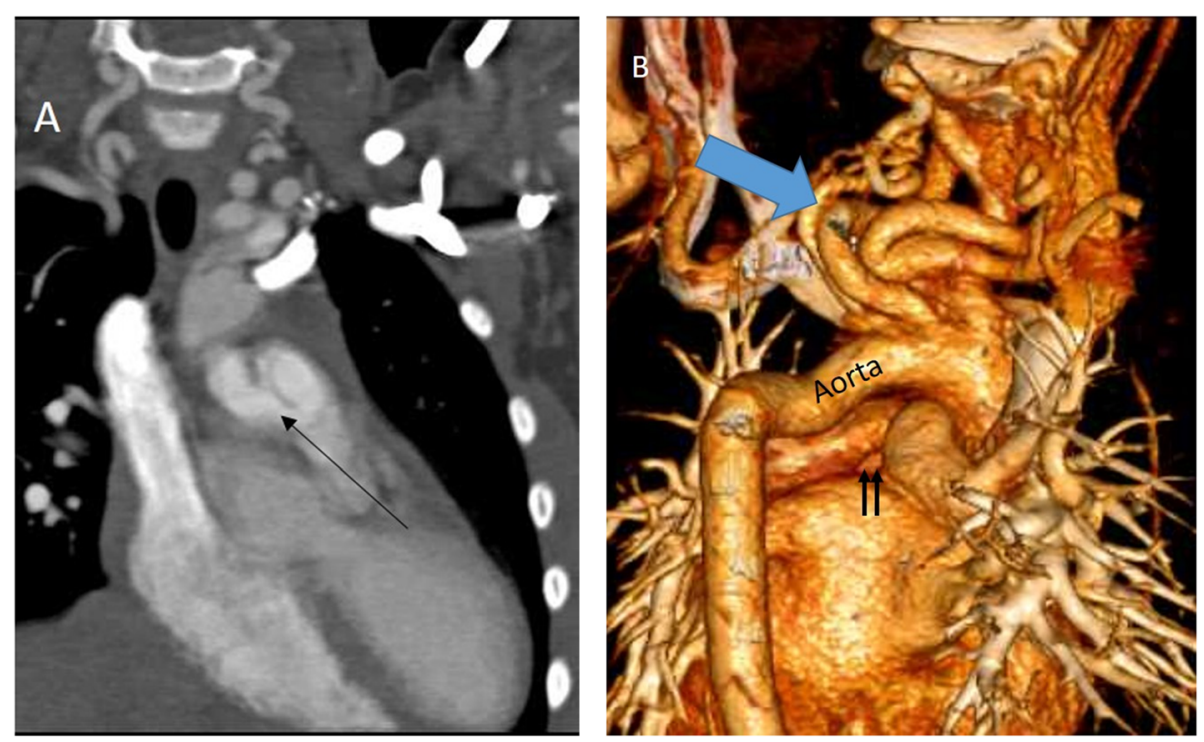

Fig. 4 A 3-year-old boy with arterial tortuosity syndrome. a Coronal CTA shows short MPA with early division into two branches (arrow). b 3DVR: elongated, tortuous aorta and great arteries (blue and black arrows) indicating arterial tortuosity syndrome. Small black arrows indicate the $\mathrm{V}$ shaped appearance of MPA due to early division 

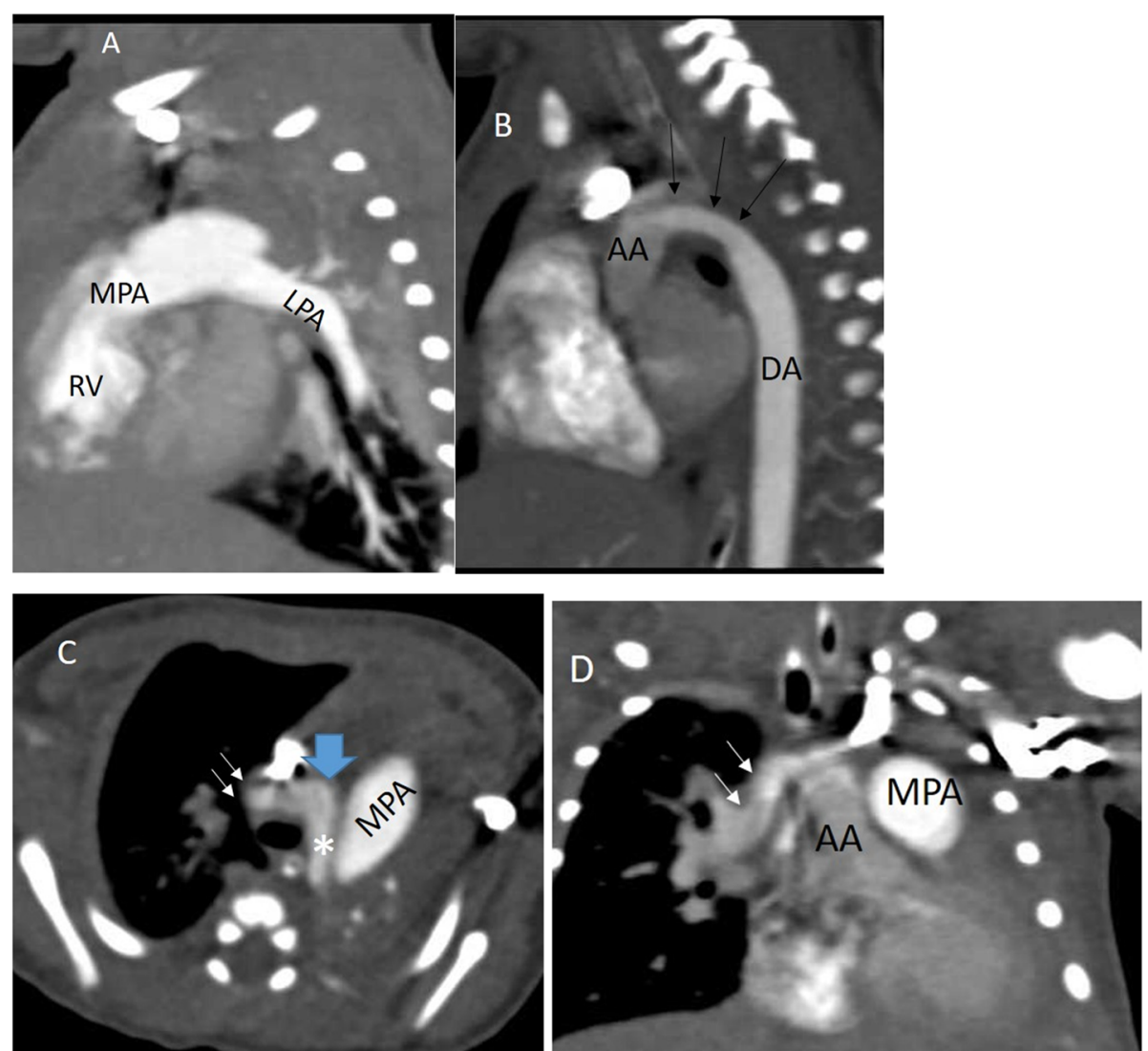

Fig. 5 A 2-week-old baby with anomalous origin of right pulmonary artery form aorta. a Sagittal CTA: dilated MPA and LPA. b Sagittal CTA: hypoplastic aortic arch (arrows). c Axial CTA shows hypoplastic aortic arch (*) and anomalous origin of RPA (arrows) from the cephalic part of ascending aorta (AA) (blue block arrow). d Coronal CTA: the RPA (arrows) arises from the cephalic part of AA

images can be used to establish accurate measurements of the luminal diameter of the pulmonary artery that correlate highly with findings at cardiac catheterization [15]. Echocardiography and cardiac catheterization consider the main modalities in the diagnostic process of complex congenital heart diseases (CHD). However, its performance may be lacking in terms of visualization of extracardiac structures such as airways abnormalities. CT is mandatory to diagnose the type of anomaly and the relationship between the trachea and the esophagus, which are surrounded by vascular structures. Three-dimensional (3D) reconstructions of trachea and vessels permit an understanding of their reciprocal relationship allowing the surgeon to plan the surgical strategy. This kind of analysis should be performed before planning surgical interventions [5]. Tracheal stenosis may not be limited to the aberrant vascular compression but due to congenital stenosis. The anesthesiologist should be aware of this point as it may pose risk during the ETT introduction. So, CT scan is not only important for surgical planning but also to alert anesthesiologists about potential complications caused by airway anomalies. In our study, the most common pulmonary artery anomalies were atresia, stenosis, and hypoplasia. Liu et al. [16] stated that pulmonary atresia is a rare anomaly with a prevalence of approximately 1 in 200,000, but in our study, it was the most common anomaly with 16 cases; it may be because our hospital is a tertiary referral. Leonard et al. [17] classified pulmonary atresia into three types, pulmonary atresia with the intact interventricular septum, pulmonary atresia with VSD, and complex pulmonary atresia. Pulmonary atresia with a ventricular septal defect is known as a group of congenital cardiovascular anomalies in which there is complete disruption between the right ventricular outflow tract and the pulmonary trunk. These patients may have atresia of part or all of the central pulmonary arteries [18]. In our study, there are 11 cases of pulmonary atresia with VSD, 4 cases of pulmonary atresia with the intact interventricular septum, and 1 case with complex pulmonary atresia, and it agrees with Leonard et al. [17]. In this study, atresia of MPA as well as RPA and LPA in 10 cases, atresia of MPA and RPA in 3 cases, and atresia of MPA and LPA in 3 cases. Associated congenital 

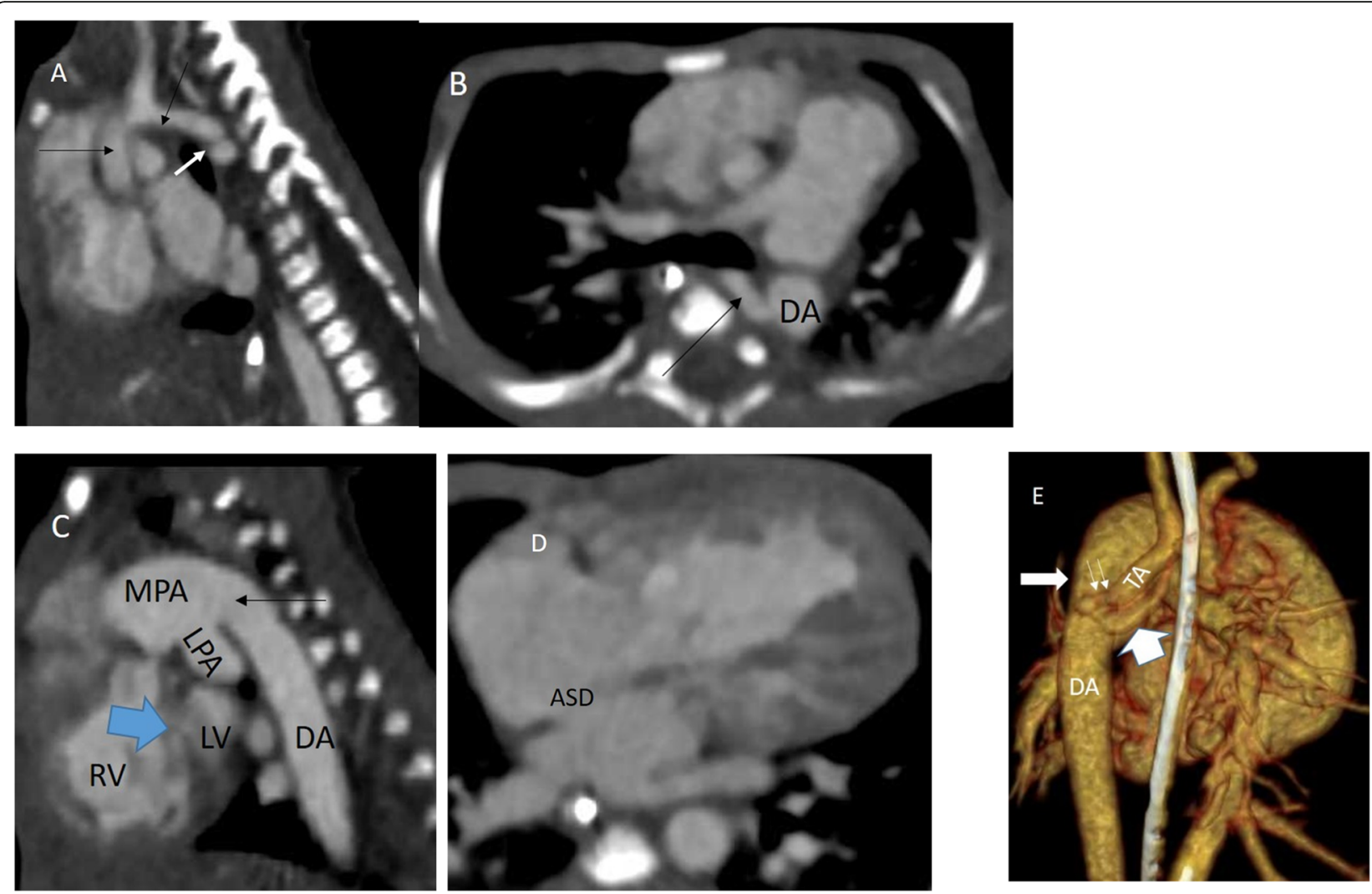

Fig. 6 A 10-day-old boy with distress and low blood pressure in bilateral lower and right upper limbs. a Sagittal CTA shows focal critical aortic coarctation (white arrow), small AA and aortic arch (black arrow). b Axial CTA: Aberrant right subclavian artery (RSCA) (arrow) arising from the cephalic part of DA distal to the coarctation. c Sagittal CTA: dilated MPA and LPA, large PDA (black arrow) supplying DA indicating duct dependent systemic circulation, VSD (blue arrow), small LV and enlarged RV. d Axial CTA: ASD and enlarged right side of heart. e 3DVR shows severe aortic coarctation (small arrows), large PDA (small block arrow), and aberrant RSCA (large black arrow) arises distal to the coarctation which explains the low BP measured from right UL

anomalies more with LPA atresia such as tetralogy of Fallot and this agree with Ryu et al. [19] that stated right pulmonary artery atresia usually occurs in the absence of other congenital abnormalities, while left pulmonary artery atresia often occurs with other congenital cardiac conditions, such as of tetralogy of Fallot. In our study, the most common associated finding with pulmonary atresia is PDA, VSD, and tetralogy of Fallot, and this is in agreement with Harikrishnan et al. [20]. In Fallot tetralogy with severe infundibular stenosis, there may be complete obstruction of pulmonary blood flow. This is known as Fallot tetralogy with pulmonary atresia and considers a specific type of pulmonary atresia with VSD. Complete disconnection of the right ventricle from the pulmonary arteries and the obligatory presence of extracardiac sources of pulmonary arterial blood flow differentiate pulmonary atresia with VSD type from the Fallot tetralogy [21]. Pulmonary artery stenosis classified into several types: type I, involving main pulmonary artery; type II, involving bifurcation; type
III, multiple peripheral stenoses; and type IV, central and peripheral stenosis [22]. In our study, stenosis of the pulmonary artery in 13 cases (25\%), 6 cases type I, 4 cases type II, 3 cases type III, and no cases type $\mathrm{IV}$, the most common type is type 1 (6 cases), and this agrees with Hirsig et al. [23]. Although pulmonary stenosis may be an isolated finding, it is often syndromic and/or associated with other cardiac abnormalities [24]. In our study, pulmonary stenosis was common apart of Fallot tetralogy (7 cases), and this agrees with Helmut et al. [25], or syndromic such as in our study arterial tortuosity syndrome (ATS) that is proved by gene testing and it is in agreement with Callewaert et al. [26] and Bhat et al. [27] that stated that it is extremely rare autosomal recessive disease caused by alterations in the SLC2A10 gene. The most common presentation in all children is tortuous artery; characteristic angiographic feature showed pulmonary stenosis, significant tortuosity of the great vessels such as aorta, changes in the caliber and significant areas of widening and narrowing are seen in 


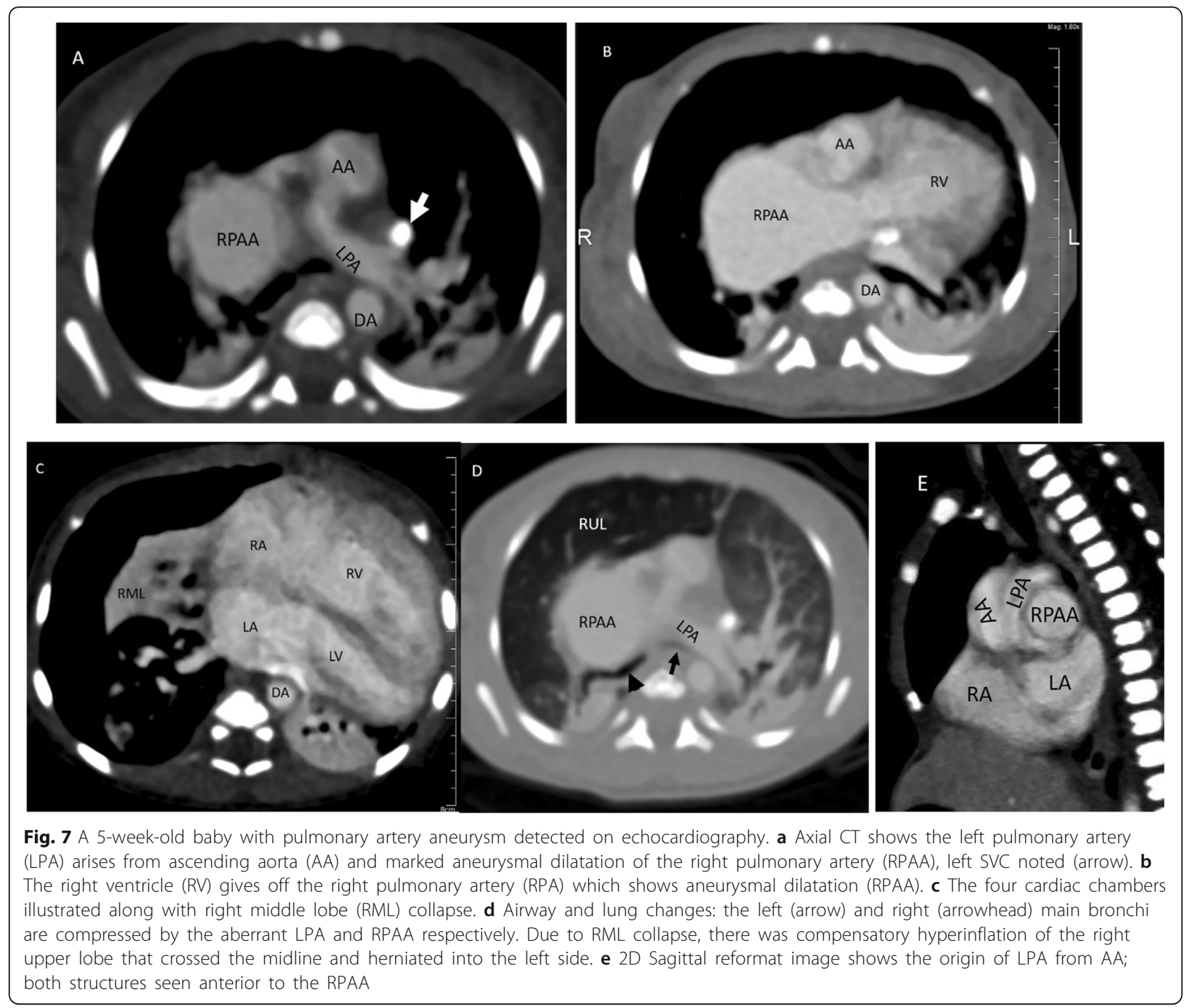

medium-sized arteries such as common carotid and subclavian arteries which also involve the abdominal vasculature, e.g., superior mesenteric artery, and all is giving a clue to the diagnosis of arterial tortuosity syndrome with other radiological and clinical finding. Hypoplasia of the pulmonary artery can be diagnosed when narrowing was diffuse involving a long segment of the artery [28]. In 10 cases, there was hypoplasia of the pulmonary artery. Pulmonary artery hypoplasia is a rare congenital anomaly. It is frequently associated with other congenital anomalies, such as tetralogy of Fallot, a ventricular septal defect, transposition of great vessels, and aortic arch anomalies [29]. In our study, there were 8 cases pulmonary artery dilatation, 6 cases dilated MPA and left pulmonary artery and 2 dilated MPA; all are associated with other multiple cardiac anomalies and it differs from idiopathic dilatation of the pulmonary trunk that it is a rare congenital anomaly comprising of pulmonary trunk enlargement with or without dilatation of the right and left pulmonary arteries. For this diagnosis, exclusion of pulmonary and cardiac diseases (mainly pulmonary valve stenosis) and confirmation of the presence of normal pressures in the right ventricle and pulmonary artery is required [30]. A group of anomalies that affects both the pulmonary artery and aorta are called aorto-pulmonary anomalies; they include truncus arteriosus, patent ductus arteriosus, and transposition of great vessels. In our study, there were 2 cases of truncus arteriosus and 27 cases patent ductus arteriosus. Truncus arteriosus accounts for $1 \%$ of congenital cardiac disease and is defined by a single great artery supplying the systemic, coronary, and pulmonary blood flow. The single truncal valve may have two to five leaflets, which may be stenotic or insufficient. An atretic pulmonary valve and a 

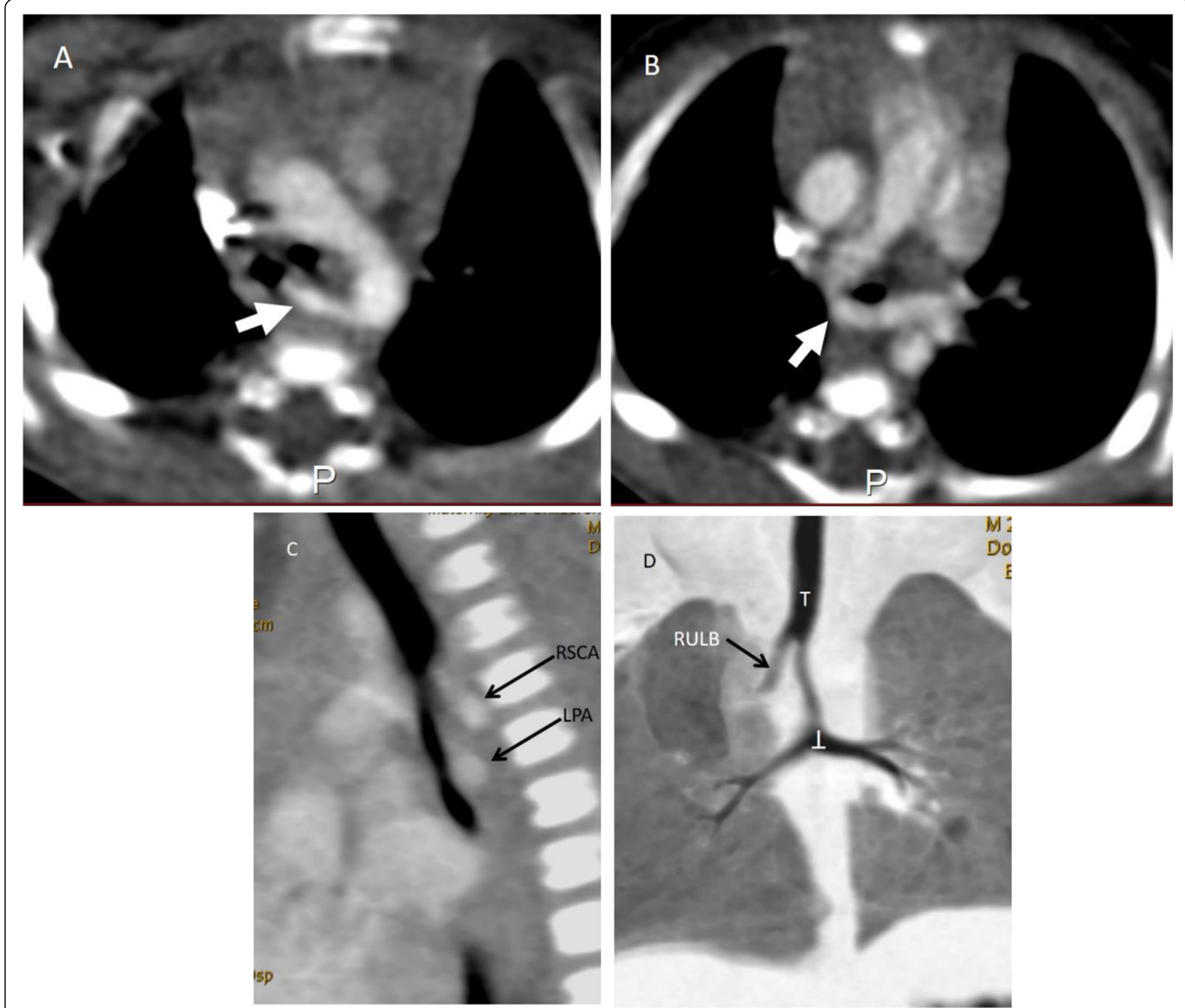

Fig. 8 An 8-day-old girl with respiratory distress. She has an aberrant right SCA (arrow in a) and aberrant left pulmonary artery that arises from the right pulmonary artery (arrow in $\mathbf{b}$ ). The aberrant LPA passes between the trachea and the esophagus. Sagittal reformat MinIP image (c) shows the aberrant RSCA and LPA as well as the long tracheal stenosis. $\mathbf{d}$ MinIP image shows right upper lobe bronchus (RULB) arising from trachea (bronchus suis). Distal to the take-off of RULB, the trachea appears narrow with low T-shaped carina (inverted T) caused by the horizontal orientation of the left main bronchus and the bronchus intermedius. Comments: Tracheal stenosis was not limited to the aberrant LPA denoting congenital stenosis rather than localized compression by the aberrant LPA. The anesthesiologist should be aware of the tracheal RULB as it poses risk during ETT introduction. So, we can also say that CT scan is not only important for surgical planning but also to alert anesthesiologists about potential complications caused by the airway anomalies

ventricular septal defect may also be present. Truncus arteriosus is rare with an incidence of in 1 in 10,000 births [31]. The classification of arteriosus devised by Van Praagh [32] are type A1: aorta and main pulmonary artery share a common arterial trunk, type A2: separate origins of the branch pulmonary arteries from the left and right lateral aspects of the common trunk, type A3: origin of one branch pulmonary artery (usually the right) from the common trunk, with other lung supplied either by collaterals or a pulmonary artery arising from the aortic arch, type A4: coexistence of an interrupted aortic arch. According to this classification, our two cases are type 1 . In our study, 5 cases have aberrant left pulmonary artery and 3 cases have aberrant right pulmonary artery. Aberrant right pulmonary artery is associated with aortic anomalies, aberrant left pulmonary artery is associated with anomalies subclavian arteries, and these agree with Kutsche et al. [33] who stated that aberrant pulmonary artery is anomalous origin of a branch pulmonary artery from ascending aorta and it is $0.3 \%$ of congenital heart disease, aberrant left pulmonary 


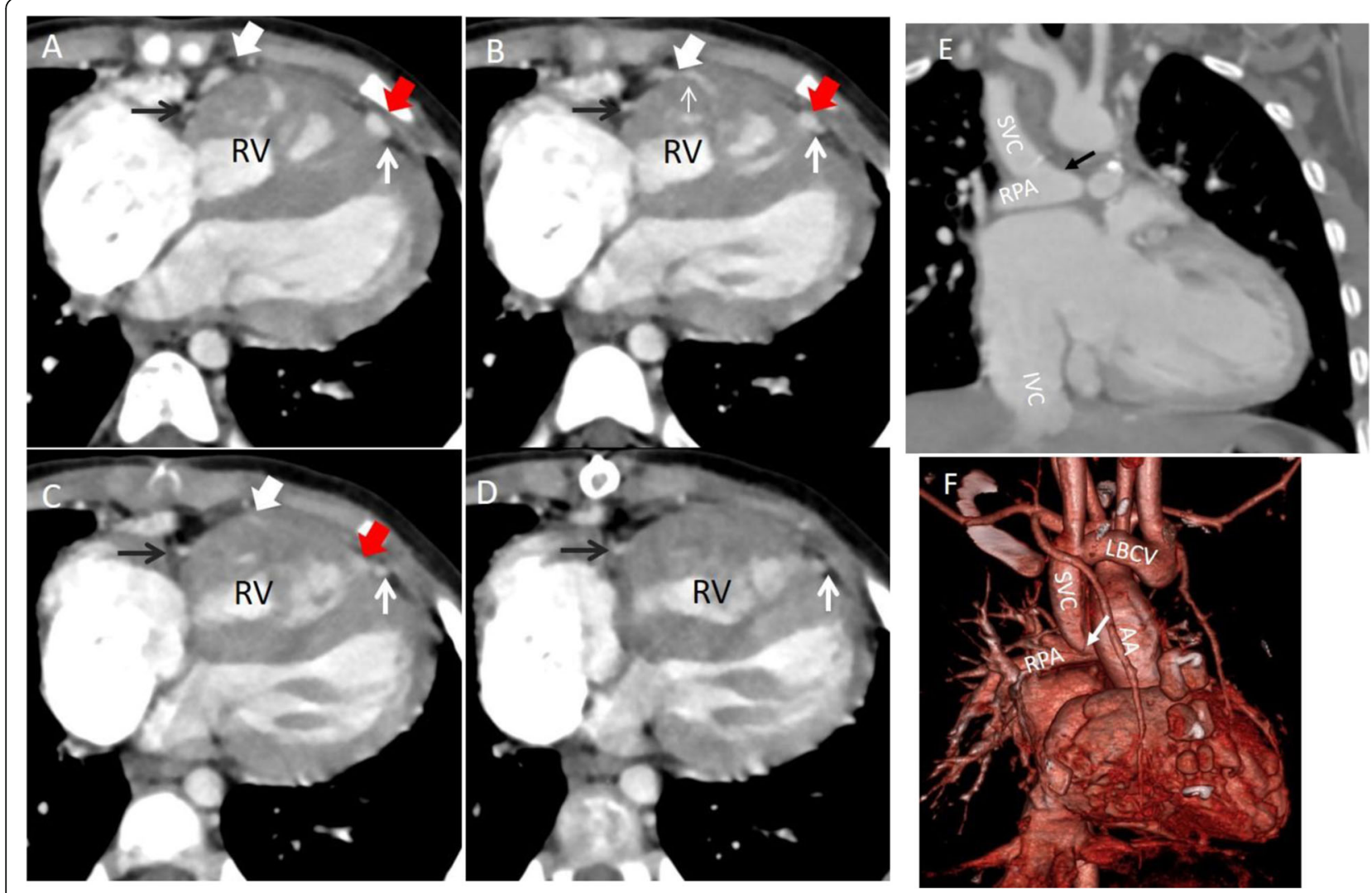

Fig. 9 3-year-old-girl with pulmonary and tricuspid atresia, post-Glenn surgery, on aspirin. She developed hemoptysis therefore CTA was requested. a-d Sequential axial CTA images show marked RV hypertrophy with fistula (block white arrow) between RV cavity and right coronary artery (RCA = black arrow). Another fistula (red and black arrows) between RV cavity and the left anterior descending artery (LAD = white arrow). The RV-RCA fistula shows focal stenosis (thin white arrow in b) whereas the RV-LAD fistula shows focal aneurysmal dilatation. e 2D coronal MIP reformat and $\mathbf{f}$ 3DVR shows patent SVC-RPA anastomosis (arrow) without stenosis

artery may be associated with tetralogy of Fallot, and anomalous subclavian arteries. On the contrary, patent ductus arteriosus and aortic anomalies may be associated with the aberrant right pulmonary artery. Left pulmonary artery sling represents an anatomical variant characterized by the left pulmonary artery arising from the right pulmonary artery and passing above the right main bronchus and in between the trachea and esophagus to reach the left lung. It may lead to compression and focal stenosis of the trachea [34]. In our study, one case of left pulmonary artery sling is detected. In our study, 2 cases of the aberrant left pulmonary artery with airway compression but one of the causes is an aberrant left pulmonary artery and the other is due to congenital stenosis so that CT scan is not only important for surgical planning but also to alert anesthesiologists about potential complications caused by the airway anomalies. Complications following cardiothoracic surgery are responsible for increased morbidity and mortality. Vascular complications in particular are significant contributors to poor patient outcomes due to either hemorrhage or thrombosis and ischemia. Evaluation of vascular complications in the postoperative patient requires a rapid and reliable imaging approach. Vascular complications after cardiothoracic surgery include pulmonary artery thrombosis, pseudoaneurysm, pulmonary vein thrombosis, vascular fistulas, stenosis, and infarction. MDCT is often the imaging modality of choice to visualize the entire cardiothoracic vasculature, airways, lung parenchyma, and mediastinum [35]. In our study, patients with pulmonary and tricuspid atresia, post-Glenn surgery, on aspirin, developed marked RV hypertrophy with a fistula between RV cavity and right coronary artery. Another fistula between the RV cavity and the left anterior descending artery. The RV-RCA fistula shows focal stenosis whereas the RV-LAD fistula shows focal aneurysmal dilatation. MDCT is a rapid and non-invasive imaging study used for the diagnosis of pediatric thoracic vasculature diseases as an alternative to conventional angiography. Three-dimensional (3D) images allow accurate delineation of vascular anomalies that can be used as a vascular road map by surgeons. 


\section{Conclusion}

MDCT scanner can be an alternative to diagnostic conventional angiography for the non-invasive assessment of the pulmonary artery. Higher quality multiplanar and 3D reconstruction achieved by the MDCT scanners offer a rapid, reliable, and non-invasive technique that can be used for the evaluation and preoperative assessment of thoracic vascular and extra-vascular anatomy in infants and children with suspected congenital heart disease. CT technologies are constantly developing collaboration between radiologists, pediatric cardiologists, and anesthesiologists, which is essential for improving CT performance.

\section{Abbreviations}

2D: Two-dimensional; 3D: Three-dimensional; AA: Ascending aorta; ASD: Atrial septal defect; BP: Blood pressure; CHD: Congenital heart diseases; CT: Computed tomography; CTA: Computed tomography angiography; ETT: Endotracheal tube; LAD: Left anterior descending artery; LPA: Left pulmonary artery; LV: Left ventricle; MAPCAs: Major aortopulmonary collateral arteries; MDCT: Multi-Detector computed tomography; MDCTA: Multidetector computed tomography angiography; MinIP: Minimum intensity projection images; MIP: Maximum intensity projection; MPA: Main pulmonary artery; MRI: Magnetic resonance imaging; PA: Pulmonary artery; PDA: Patent ductus arteriosus; RCA: Right coronary artery; RML: Right middle lobe; RPA: Right pulmonary artery; RPA: Right pulmonary artery; RPAA: Right pulmonary aneurysmal dilatation; RSCA: Right subclavian artery; RV: Right ventricle; TA: Transverse aorta; TOF: Tetralogy of Fallot; TTE: Transthoracic echocardiogram; UL: Upper limb; VR: Volume rendering; VSD: Ventricular septal defect

\section{Acknowledgements}

Not applicable.

\section{Authors' contributions}

$\mathrm{MMH}$ carried out the radiological studies, participated in the design of the study, gave the idea, collected the patients' data, and performed the statistical analysis. AHA participated in the sequence alignment and drafted the manuscript. KAA participated in the acquisition of data. HAS participated in the sequence alignment. SMA participated in the design of the study and performed the statistical analysis. GA conceived of the study, and participated in its design and coordination and helped to draft the manuscript. MMR wrote the paper with revision. All authors read and approved the final manuscript.

\section{Funding}

This study had no funding from any resource.

\section{Availability of data and materials}

The datasets used and/or analyzed during the current study are available from the corresponding author on reasonable request.

\section{Ethics approval and consent to participate}

This study was approved by the Research Ethics Committee of maternity and children hospital in Saudi Arabia. The patients were less than 16 years old at the time of the study, and written informed consent for their participation was given by their parent or legal guardian. The committee's reference number is not available.

\section{Consent for publication}

All patients were less than 16 years old, and written informed consent for the publication of this data was given by their parent or legal guardian.

\section{Competing interests}

The authors declare that they have no competing interests.

\section{Author details}

${ }^{1}$ Radiology Department, Mansoura University Hospitals, Mansoura, Egypt.

${ }^{2}$ Radiology Department, Zagazig University, Zagazig, Egypt. ${ }^{3}$ Maternity and Children Hospital, Holy Makkah, Kingdom of Saudi Arabia.

Received: 7 October 2019 Accepted: 27 November 2019

Published online: 18 December 2019

\section{References}

1. Gaca AM, Jaggers JJ, Dudley LT et al (2008) Repair of congenital heart disease: a primer. Part 1. Radiology 247:617-631

2. Tsai IC, Chen MC, Jan SL et al (2008) Neonatal cardiac multidetector row CT: why and how we do it. Pediatr Radiol 38:438-451

3. Leschka S, Oechslin E, Husmann L et al (2007) Pre- and postoperative evaluation of congenital heart disease in children and adults with 64section CT. RadioGraphics 27:829-846

4. Hollingsworth $C L$, Yoshizumi TT, Frush DP et al (2007) Pediatric cardiacgated $C T$ angiography: assessment of radiation dose. AJR 189:12-18

5. Mehta R, Lee KJ, Chaturvedi R et al (2008) Complications of pediatric cardiac catheterization: a review in the current era. Catheter Cardiovasc Interv 72: 278-285

6. Taylor AM (2008) Cardiac imaging: MR or CT? Which to use when. Pediatr Radiol 38:433-\$438

7. Bean MJ, Pannu H, Fishman EK (2005) Three-dimensional computed tomographic imaging of complex congenital cardiovascular abnormalities. J Comput Assist Tomogr 29:721-724

8. Frush DP, Herlong JR (2005) Pediatric thoracic CT angiography. Pediatr Radiol 35:11-25

9. Gilkeson RC, Ciancibello L, Zahka K (2003) Multidetector CT evaluation of congenital heart disease in pediatric and adult patients. AJR 180: 973-980

10. Goo HW, Park IS, Ko JK et al (2003) CT of congenital heart disease: normal anatomy and typical pathologic conditions. RadioGraphics 23: 147-165

11. Siegel MJ (2003) Multiplanar and three-dimensional multi-detector row CT of thoracic vessels and airways in the pediatric population. Radiology 229:641-650

12. Spevak PJ, Johnson PT, Fishman EK (2008) Surgically corrected congenital heart disease: utility of 64MDCT. AJR 191:854-861

13. Westra SJ, Hill JA, Alejos JC et al (1999) Three-dimensional helical CT of pulmonary arteries in infants and children with congenital heart disease. AJR 173:109-115

14. Greil GF, Schoebinger M, Kuettner A et al (2006) Imaging of aortopulmonary collateral arteries with high-resolution multidetector $\mathrm{CT}$. Pediatr Radiol 36: 502-509

15. Ley S, Zaporozhan J, Arnold R et al (2007) Preoperative assessment and follow-up of congenital abnormalities of the pulmonary arteries using CT and MRI. Eur Radiol 17:151-162

16. Liu B, Monroe EJ, Kogut MJ et al (2015) Proximal interruption of the pulmonary artery: transcatheter embolization for emergent management of massive hemoptysis. Radiol Case Rep 8(3):865

17. Leonard H, Derrick G, O'Sullivan J et al (2000) Natural and unnatural history of pulmonary atresia. Heart 84:499-503

18. O'Leary PW, Edwards WD, Julsrud PR et al (2008) Pulmonary atresia and ventricular septal defect. In: Allen HD, Driscoll DJ, Shaddy RE, Feltes TF (eds) Moss and Adam's heart disease in infants, children, and adolescents. Lippincott Williams \& Wilkins, Baltimore, pp 878-888

19. Ryu DS, Spirn PW, Trotman-Dickenson B et al (2004) HRCT findings of proximal interruption of the right pulmonary artery. J Thorac Imaging 19: $171-175$

20. Harikrishnan S, Tharakan J, Titus T et al (2000) Central pulmonary artery anatomy in right ventricular outflow tract obstructions. Int J Cardiol 73: 225-230

21. Tchervenkov Cl, Roy N (2000) Congenital Heart Surgery Nomenclature and Database Project: pulmonary atresia-ventricular septal defect. Ann Thorac Surg 69:97-105

22. Weissleder R (2003) Primer of diagnostic imaging, 3rd edn. Philadelphia: Mosby

23. Hirsig LE, Sharma PG, Verma $\mathrm{N}$ et al (2018) Congenital pulmonary artery anomalies: a review and approach to classification. J Clin Imaging Sci 8:29 
24. Beçu L, Somerville J, Gallo A (1976) 'Isolated' pulmonary valve stenosis as part of more widespread cardiovascular disease. Br Heart J 38:472-482

25. Helmut B, Judy H, Bo J et al (2009) Echocardiographic assessment of valve stenosis: EAE/ASE recommendations for clinical practice. Eur J Echocardiogr 10(1):1-25

26. Callewaert B, De Paepe A, Coucke P (2014) Arterial tortuosity syndrome. In: Adam MP, Ardinger HH, Pagon RA et al (eds) GeneReviews ${ }^{\oplus}$. University of Washington, Seattle, Seattle 1993-2019. Available from: https://www.ncbi. nlm.nih.gov/books/NBK253404/

27. Bhat V (2009) Arterial tortuosity syndrome: an approach through imaging perspective. J Clin Imaging Sci 4:44

28. Yildirim A, Karabulut N, Dogan S et al (2011) Congenital thoracic arterial anomalies in adults: a CT overview. Diagn Interv Radiol 17:352-362

29. Kadir IS, Thekudan J, Dheodar A et al (2002) Congenital unilateral pulmonary artery agenesis and as-pergilloma. Ann Thorac Surg 74:21692171

30. Castañer E, Gallardo X, Rimola J et al (2006) Congenital and acquired pulmonary artery anomalies in the adult: radiologic overview. Radiographics 26(2):349-371

31. Kimura-Hayama ET, Meléndez G, Mendizábal AL et al (2010) Uncommon congenital and acquired aortic diseases: role of multidetector $C T$ angiography. Radiographics 30:79-98

32. Van Praagh R, Van Praagh S (1965) The anatomy of common aorticopulmonary trunk (truncus arteriosus communis) and its embryologic implications. Am J Cardiol 16(3):406-425

33. Kutsche LM, Van Mierop LH (1988) Anomalous origin of a pulmonary artery from the ascending aorta: associated anomalies and pathogenesis. Am J Cardiol 61:850-856

34. Ganesh V, Hoey ET, Gopalan D (2009) Pulmonary artery sling: an unexpected finding on cardiac multidetector CT. Postgrad Med J 85(1001):128

35. Restrepo CS, Vargas D, Martinez-Jimenez S et al (2018) Post-operative imaging of pulmonary vessels. Cardiovasc Diagn Ther 8(3):362-371

\section{Publisher's Note}

Springer Nature remains neutral with regard to jurisdictional claims in published maps and institutional affiliations.

\section{Submit your manuscript to a SpringerOpen ${ }^{\circ}$ journal and benefit from:}

- Convenient online submission

- Rigorous peer review

- Open access: articles freely available online

High visibility within the field

- Retaining the copyright to your article

Submit your next manuscript at $\boldsymbol{\nabla}$ springeropen.com 